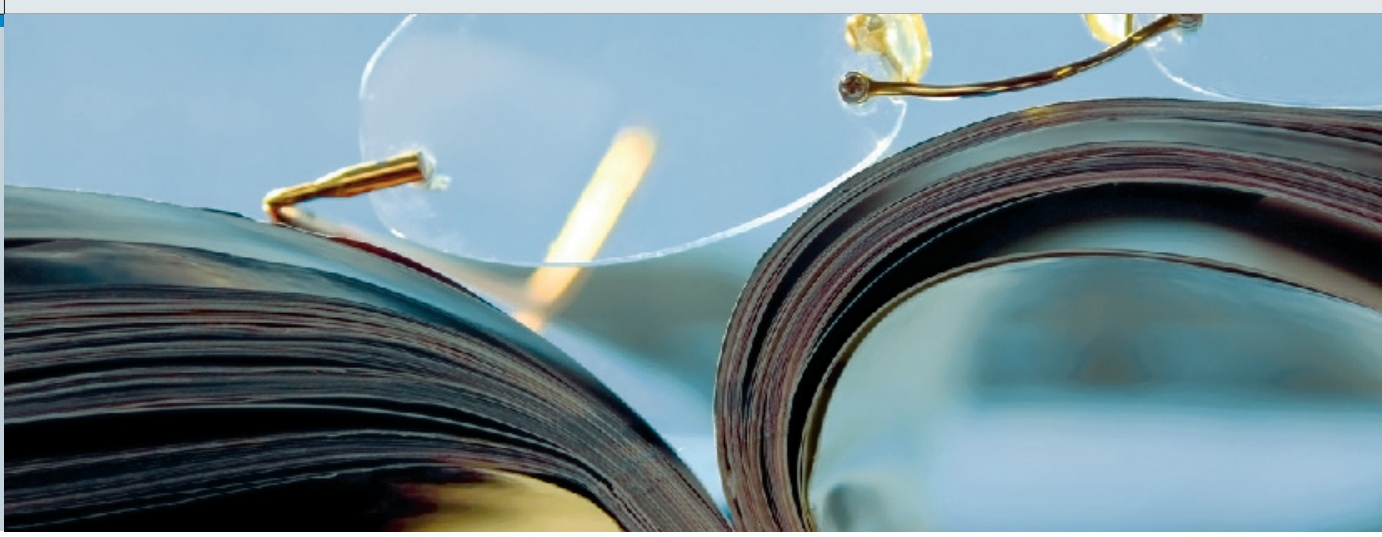

\section{Neue Klassifikation der vestibulären Symptome}

\author{
In einem Konsensuspapier der Bárány-Gesellschaft sind vestibuläre \\ Beschwerden in einer neuartigen Systematik zusammengefasst worden.
}

$\mathrm{D}$ ie Autoren konzentrierten sich in ihrer Systematik strikt auf Symptome ohne Verbindung zur Pathogenese oder den zugrunde liegenden Erkrankungen. In der Nomenklatur werden in einer ersten Ebene vier Entitäten definiert:

1. Schwindel (,vertigo") oder auch ,interner Schwindel" als pathologische Empfindung einer real nicht stattfindenden Bewegung im Raum,

2. Unsicherheit bzw. Einschränkung in der Raumorientierung („dizziness"), hier definiert als Störung ohne Bewegungswahrnehmung,

3. vestibulär-visuelle Symptome („,vestibulo-visual symptoms") und

4. Gleichgewichtsstörungen („postural symptoms") als Instabilität beim Gehen, Stehen oder Sitzen.

Das Symptom Schwindel wird weiter in eine spontane und getriggerte Form eingeteilt. Bei letzterer werden sieben verschiedene auslösende Faktoren unterschieden: Lagerung, Kopfbewegung, visuell induziert, akustisch induziert, durch Valsalva-Manöver ausgelöst, orthostatisch bedingt sowie „andere“ Triggerfaktoren. Für das Hauptsymptom Unsicherheit in der Raumorientierung haben die Autoren die gleiche Untergliederung in „spontan“ und "getriggert" sowie bei der getriggerten Form wiederum in die analogen sieben Unterformen wie beim Schwindel vorgenommen. Die visuellen Symptome werden unterteilt in: „externer Schwindel“ (falsche visuelle Empfindung einer sich drehenden oder bewegenden Umwelt), Oszillopsie (fixierte Objekte werden als schwankend, wackelnd oder zitternd wahrgenommen), visuelle Verzögerung (die visuelle Wahrnehmung bei der eigenen Kopfbewegung erfolgt mit Zeitverzögerung), visuelle Schieflage (Wahrnehmung einer Umwelt mit Abweichung von der vertikalen Achse) und verschwommenes Sehen während oder kurz nach Kopfdrehung. Bei den Gleichgewichtsstörungen existieren vier Unterformen: Gefühl der instabilen Position, der Instabilität mit Drehen oder Neigen in eine Richtung, gleichgewichtsbedingte Fallneigung (noch ohne Sturz) sowie gleichgewichtsbedingte Stürze. In der Nomenklatur erfolgen noch zusätzliche Differenzierungen wie etwa Drehschwindel und Nicht-Drehschwindel bei „vertigo" oder persistierende und transiente Formen. Dr. Andreas Fischer

\section{Bisdorff A et al. Classification of vestibular} symptoms: towards an international classification of vestibular disorders. J Vestib Res 2009; 19: $1-13$

Kommentar: Die Bárány-Gesellschaft hat 2006 ein Kommittee ins Leben gerufen, das nicht nur zur Freude der Vestibularis-Forscher (endlich) zum Ziel hat, eine Systematik zur Klassifikation und damit auch eine verbindliche Nomenklatur der Gleichgewichtsstörungen zu erarbeiten. Das erste Konsensuspapier liegt nun unter der Bezeichnung ICVD-I (international classification of vestibular disorders) classification of symptoms 1.0 vor. Diese Aufgabe war nicht nur überfällig, weil es überlappende, schwammige Bezeichnungen für Gleichgewichtsstörungen gibt, sondern auch schwierig, weil es auch in den verschiedenen Sprach- und Kulturräumen Unterschiede in der sprachlichen Umschreibung von Schwindelformen gibt. Zudem sind auch die Nachbardisziplinen wie die Neurologie, Neuropsychiatrie und Ophthalmologie zu berücksichtigen.

Nach ICVD-I werden nun 1. "Vertigo" als Drehschwindel von 2. „Dizziness" als Unsicherheit, "Nicht-Drehschwindel“, 3. optokinetischer Schwindel und 4. Gleichgewichtsstörungen („postural symptoms") unterschieden. Diese werden wiederum in "spontane" und ",getriggerte", vorübergehende und dauernde und weitere Untergruppen aufgeteilt. Daraus ergibt sich ein Kodierungsalgorithmus, der auf den ersten Blick sehr übersichtlich und sinnvoll erscheint. Die Schematik scheint geeignet, mehr Ordnung in Schwindeldiagnostik und -statistik bringen zu können. Das sollte bei konsequenter Anwendung Untersuchungen und Behandlungen weltweit vergleichbarer werden lassen. Von der Initiative der Bárány-Gesellschaft ist eine normative Kraft zu erwarten, denn ihr gehören führende Vestibularis-Forscher an. Daher ist davon auszugehen, dass sich auch "normale“ HNO-Ärzte nachhaltig über diesen Fortschritt freuen können, wenn Klassifikation und Nomenklaturvorschläge auch allgemeinen Konsens finden.

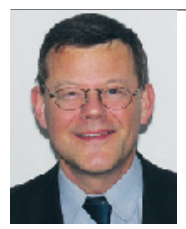

Prof. Dr. med. Olaf Michel Universitair Ziekenhuis, Brüssel E-Mail:OMichel@uzbrussel.be 